\title{
Acoustic and Electromagnetic Wave Interaction: Estimation of Doppler Spectrum From an Acoustically Vibrated Metallic Circular Cylinder
}

Kamal Sarabandi, Fellow, IEEE, and Daniel E. Lawrence, Member, IEEE

\begin{abstract}
The idea of using acoustically induced Doppler spectra as a means for target detection and identification is introduced. An analytical solution for the calculation of the bistatic scattered Doppler spectrum from an acoustically excited, vibrating metallic circular cylinder is presented. First the electromagnetic scattering solution of a slightly deformed circular cylinder is obtained using a perturbation method. Then, assuming the vibration frequency is much smaller than the frequency of the incident electromagnetic wave, a closed form expression for the time-frequency response of the bistatic scattered field is obtained which can be used directly for estimating the Doppler spectrum. The acoustic scattering solution for an incident acoustic plane wave upon a solid elastic cylinder is applied to give the displacement of the cylinder surface as a function of time. Results indicate that the scattered Doppler frequencies correspond to the mechanical vibration frequencies of the cylinder, and the sidelobe Doppler spectrum level is, to the first order, linearly proportional to the degree of deformation and is a function of bistatic angle. Moreover, the deformation in the cylinder, and thus the Doppler sidelobe level, only becomes sizeable near frequencies of normal modes of free vibration in the cylinder. Utilizing the information in the scattered Doppler spectrum could provide an effective means of buried object identification, where acoustic waves are used to excite the mechanical resonances of a buried object.
\end{abstract}

Index Terms-Acoustics, cylinder, Doppler spectrum, electromagnetic scattering.

\section{INTRODUCTION}

G ROUND penetrating radars have been proposed for detection and identification of buried objects [1]. For civilian applications such radars are needed to locate underground pipes, conduits, and cables, while military applications include mine detection and clearing abandoned military practice ranges of unexploded ordinance (UXO). Over the past three decades much effort has been devoted to the development of hardware and advanced detection algorithms to improve the performance of ground penetrating radars in so far as the probability of detection and the false alarm rate are concerned. The detectability of a buried object is severely limited by the high attenuation rate of microwaves propagating in most soil media as well as the relatively large impedance mismatch at the interface between the air and ground. Hence, the initial optimism has not yet led to the

Manuscript received April 13, 1999; revised April 19, 2002

The authors are with the Radiation Laboratory, Department of Electrical Engineering and Computer Science, University of Michigan, Ann Arbor, MI 48109-2122 USA.

Digital Object Identifier 10.1109/TAP.2003.813606

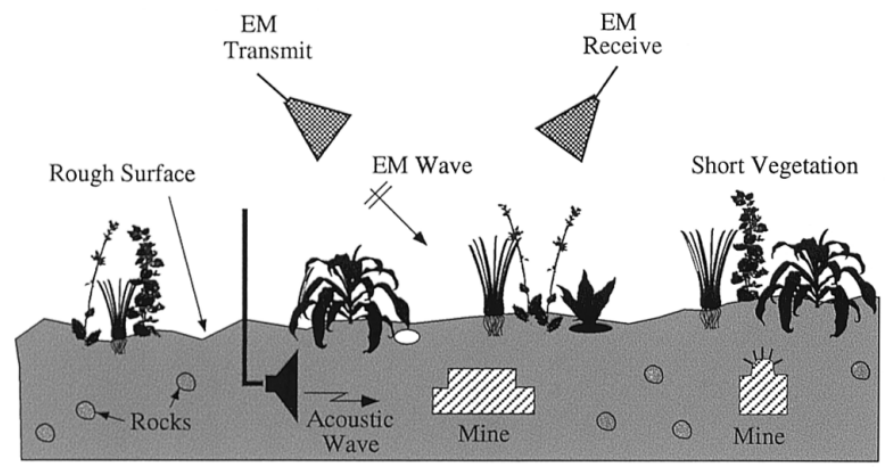

Fig. 1. Example scenario for acousto-electromagnetic object detection.

development of a reliable system that can perform satisfactorily even with the most lax false alarm rate requirements.

To increase the probability of detection without increasing the false alarm rate, measurable quantities which are characteristic of the objects of interest should be exploited. One such quantity is the electromagnetic Doppler spectrum that is scattered from a buried object being mechanically vibrated by an incident acoustic wave. If the buried objects have unique and identifiable Doppler spectra, corresponding to unique mechanical modes of the objects, then improvement in detection and identification of the objects is possible. Combining electromagnetic scattering with acoustic excitation to detect buried objects has been addressed previously [2], [3]. Recently, this idea has been examined to some extent in [4] where a radar is used to measure displacements of the earth's surface caused by a traveling acoustic wave. In this approach, object detection is based on the small changes in ground displacement when a buried object is introduced. To aid in clutter rejection, distinguishing characteristics of the buried objects of interest, such as the normal modes of vibration, should be incorporated into the detection scheme. In this paper, the application of the size and material dependent bistatic Doppler spectrum in target identification is examined. Fig. 1 shows the concept for the proposed mine detection and identification scheme.

Before an acousto-electromagnetic technique can be effective in this way, the relationship between the mechanical vibration modes of an object and the scattered electromagnetic Doppler spectrum must be understood. Perhaps the simplest scattering geometry for which an analytical solution exists is an infinitely long, circular cylinder. As a feasibility study, we will examine, analytically, the Doppler scattering from a vibrating metallic 
circular cylinder in a homogeneous surrounding medium. Admittedly, the solid metallic cylinder considered here is not the most realistic model for a landmine. Furthermore, as will be shown, the solid cylinder requires an enormous amount of acoustic power to see the effect of vibrations in the EM Doppler spectrum. The model was chosen, not for its direct applicability, but rather for its mathematical tractability. The cylinder is useful for illustrating that the mechanical resonance behavior of objects can be seen in the scattered EM Doppler spectrum.

A cylinder under mechanical vibration can be thought of as a deformed cylinder whose shape is varied with time. Hence an analytical solution for the scattered field of an arbitrary deformed cylinder is needed. Since the deformations are small, an analytical perturbation technique is chosen to calculate the scattered fields. Perturbation techniques can provide analytical solutions to problems when the surface irregularity is small and exact solutions exist for the unperturbed problems. Perturbation theory is an established analytical approach for scattering solutions and was applied by Rayleigh [5] and Maxwell [6] to certain scalar field problems. These methods have found application in electromagnetic scattering from rough surfaces [7], [8], cylinders [9]-[11] and spheres [12], [13]. The perturbation solution developed here is based on the eigenfunction expansion of vector fields in cylindrical coordinates. Yeh [10] presents a methodology for this approach and derives coefficients for the specific case of an elliptic cylinder with small eccentricity. For the present work, however, the time-varying perturbation coefficients for an arbitrarily deformed cylinder are needed and will be derived using a similar approach. Once the electromagnetic scattering from a time-varying deformed cylinder is obtained, a Fourier transform can be used to extract the Doppler spectrum. The time-varying cylinder deformation is obtained from the analytical solution for the acoustic scattering from a solid elastic cylinder. As will be shown, the bistatic Doppler spectrum may be used as a tool for noninvasive measuring of different mechanical vibration modes. A number of examples are presented to illustrate the relationship between the mechanical modes of the cylinder and the Doppler spectrum.

As a point of note, the authors have extended the present work to consider Doppler scattering from a vibrating dielectric cylinder. Results of this derived work have already been published in [14], and the interested reader is referred there.

\section{Electromagnetic ScAtTering From A Perturbed Circular CYlinder}

In this section, the bistatic scattered field from a perfectly conducting circular cylinder with arbitrary deformation and illuminated by an electromagnetic plane wave is formulated. An $e^{j \omega e t}$ time dependence for electromagnetic waves and an $e^{j \omega_{a} t}$ time dependence for acoustic waves is understood in all expressions representing waves and is suppressed.

\section{A. TM Case}

Consider an incident TM plane wave impinging upon a perfectly conducting, slightly deformed circular cylinder as shown in Fig. 2. For a vibrating cylinder whose cross section shape is

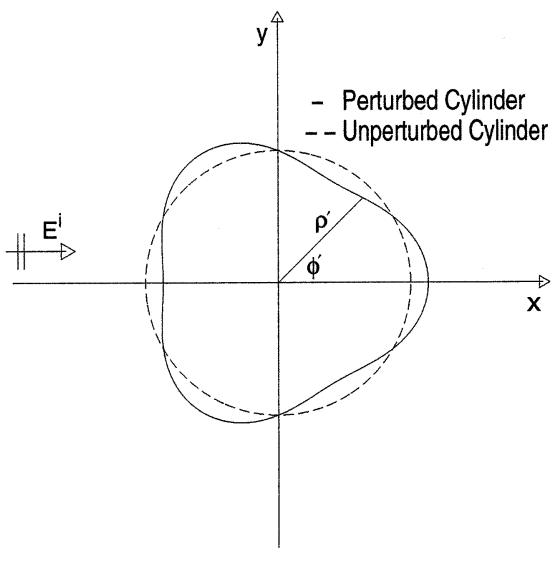

Fig. 2. Geometry for electromagnetic scattering from a perturbed cylinder.

varying with time, the scattered field will be time-varying which gives rise to the scattered Doppler spectrum. The perimeter of the cylinder's surface can be expressed in polar coordinates as

$$
\rho^{\prime}=a+b f\left(\phi^{\prime}, t\right)
$$

where $a$ is the unperturbed cylinder radius, $f\left(\phi^{\prime}, t\right)$ is a periodic and smooth function of $\phi^{\prime}$, and $b$ is a perturbation constant assumed to be much smaller than the wavelength. The standard method for the computation of the scattered field from a circular cylinder is the eigen-function expansion of the total field. Following the development in [15], the incident plane wave propagating along the $\mathrm{x}$-direction with a polarization parallel to the cylinder's axis can be expressed as

$$
E_{z}^{i}=e^{-j k \rho \cos \phi}=\sum_{n=-\infty}^{\infty}(-j)^{n} J_{n}(k \rho) e^{j n \phi}
$$

where $k$ is the wavenumber. When there is no perturbation on the surface (i.e., $b=0$ ), the scattered field is given by

$$
E_{z}^{s}=\sum_{n=-\infty}^{\infty}(-j)^{n} \frac{J_{n}(k a)}{H_{n}^{(2)}(k a)} H_{n}^{(2)}(k \rho) e^{j n \phi} .
$$

If a small perturbation is introduced on the boundary, the scattered field may be expanded in terms of a perturbation series in $k b$. To the first order in $k b$, we may write

$$
E_{z}^{s}=\sum_{n=-\infty}^{\infty}(-j)^{n} \frac{J_{n}(k a)}{H_{n}^{(2)}(k a)}\left(1+C_{n} k b\right) H_{n}^{(2)}(k \rho) e^{j n \phi}
$$

where the $C_{n}$ 's are unknown coefficients to be determined using the boundary condition $E_{z}^{i}+E_{z}^{s}=0$ on the surface of the perturbed cylinder. This expression is, of course, a permissible solution of Maxwell's equations since it satisfies both the wave equation and the radiation condition. Using Taylor series expansion, the eigen-functions in (2) and (4) can also be approximated to the first order in $k b$, i.e.,

$$
Z_{n}\left(k \rho^{\prime}\right)=Z_{n}\left(k\left(a+b f\left(\phi^{\prime}, t\right)\right) \approx Z_{n}(k a)+k b f\left(\phi^{\prime}, t\right) Z_{n}^{\prime}(k a)\right.
$$


where $Z_{n}$ represents the Bessel or Hankel function of $n$th order. Applying the boundary condition at the surface of the cylinder

$$
\begin{aligned}
& \sum_{n=-\infty}^{\infty}(-j)^{n}\left[J_{n}(k a)+k b f\left(\phi^{\prime}, t\right) J_{n}^{\prime}(k a)\right] e^{j n \phi^{\prime}}=\sum_{n=-\infty}^{\infty}(-j)^{n} \\
& \frac{J_{n}(k a)}{H_{n}^{(2)}(k a)}\left(1+C_{n} k b\right)\left[H_{n}^{(2)}(k a)+k b f\left(\phi^{\prime}, t\right) H_{n}^{(2) \prime}(k a)\right] e^{j n \phi^{\prime}} .
\end{aligned}
$$

Neglecting the $(k b)^{2}$ term and using the Wronskian relationship for Bessel functions results in the following expression:

$$
\sum_{n=-\infty}^{\infty}(-j)^{n} C_{n} J_{n}(k a) e^{j n \phi^{\prime}}=\frac{2 j f\left(\phi^{\prime}, t\right)}{\pi k a} \sum_{n=-\infty}^{\infty}(-j)^{n} \frac{e^{j n \phi^{\prime}}}{H_{n}^{(2)}(k a)} .
$$

Now if $f\left(\phi^{\prime}, t\right)$ has a Fourier series expansion with respect to $\phi^{\prime}$

$$
f\left(\phi^{\prime}, t\right)=\sum_{m=-\infty}^{\infty} A_{m}(t) e^{j m \phi^{\prime}}
$$

and is inserted into (7), the resulting solution for the coefficients, $C_{n}$, is

$$
C_{n}(t)=\frac{2(j)^{n+1}}{\pi k a J_{n}(k a)} \sum_{p=-\infty}^{\infty}(-j)^{p} \frac{A_{n-p}(t)}{H_{p}^{(2)}(k a)} .
$$

Hence for a given displacement function, $f\left(\phi^{\prime}, t\right)$, the $C_{n}$ 's calculated from (9) can be used in (4) to yield the bistatic scattered field at any angle $\phi$. In this derivation it is implicitly assumed that the time rate of variations of $f\left(\phi^{\prime}, t\right)$ is much slower than the electromagnetic frequency so that relativistic effects can be neglected. Under this assumption, a Fourier transform of the time-varying scattered field will provide the scattered Doppler spectrum.

\section{B. TE Case}

Using the same procedure, a solution for TE incidence is also possible. The magnetic field of an incident plane wave propagating in the $\mathrm{x}$-direction with polarization transverse to the cylinder's axis can be expressed as

$$
H_{z}^{i}=e^{-j k x}=\sum_{n=-\infty}^{\infty} j^{-n} J_{n}(k \rho) e^{j n \phi} .
$$

When there is no perturbation on the surface of the cylinder, the scattered field is given by

$$
H_{z}^{s}=-\sum_{n=-\infty}^{\infty} j^{-n} \frac{J_{n}^{\prime}(k a)}{H_{n}^{(2) \prime}(k a)} H_{n}^{(2)}(k \rho) e^{j n \phi} .
$$

As before, a slight perturbation is introduced on the boundary, and a perturbation series may be written for the scattered field. To the first order in $k b$, the scattered magnetic field may be written as

$$
H_{z}^{s}=-\sum_{n=-\infty}^{\infty} j^{-n} \frac{J_{n}^{\prime}(k a)}{H_{n}^{(2) \prime}(k a)}\left(1+k b D_{n}\right) H_{n}^{(2)}(k \rho) e^{j n \phi}
$$

in which the $D_{n}$ 's are found from the boundary condition, $E_{\text {tan }}^{i}+E_{\text {tan }}^{s}=0$. Where $E_{\text {tan }}$ denotes the tangent electric field at the cylinder boundary. In the TM case, the z-directed electric field is always tangent to the cylinder surface despite any perturbation in the boundary, and in the TE case for a perfect cylinder, the $\phi$ component of the electric field is always tangent to the cylinder boundary. However, when there is a perturbation, applying the boundary condition is slightly more complicated because the $\phi$ component of the electric field is no longer tangent to the boundary. To properly apply the boundary condition, the unit vector tangent to the surface must be defined and the electric field component in this direction has to be found. The unit vector tangent to the perturbed cylinder's surface is given by

$$
\hat{u}_{t}=\alpha\left[\beta \hat{u}_{\rho}+\hat{u}_{\phi}\right]
$$

where $\alpha=\left[\left(\left(b f^{\prime}\left(\phi^{\prime}, t\right)\right) /\left(a+b f\left(\phi^{\prime}, t\right)\right)\right)^{2}+1\right]^{-1 / 2}, \beta=$ $\left(\left(b f^{\prime}\left(\phi^{\prime}, t\right)\right) /\left(a+b f\left(\phi^{\prime}, t\right)\right)\right)$, and $f^{\prime}\left(\phi^{\prime}, t\right)$ is the partial derivative of $f\left(\phi^{\prime}, t\right)$ with respect to $\phi^{\prime}$. With this expression for the tangent unit vector, the tangent electric fields at the cylinder surface are found from

$E_{\text {tan }}^{i}=\frac{1}{j \omega_{e} \epsilon}\left(\nabla \times H_{z}^{i} \hat{u}_{z}\right) \cdot \hat{u}_{t}$ and $E_{\text {tan }}^{s}=\frac{1}{j \omega_{e} \epsilon}\left(\nabla \times H_{z}^{s} \hat{u}_{z}\right) \cdot \hat{u}_{t}$

Using (10) and (13) in (14) gives the incident tangent electric field evaluated at the surface

$$
E_{\text {tan }}^{i}=\frac{1}{j \omega_{e} \epsilon} \sum_{n=-\infty}^{\infty} j^{-n} \alpha\left[\beta \frac{j n}{\rho^{\prime}} J_{n}\left(k \rho^{\prime}\right)-k J_{n}^{\prime}\left(k \rho^{\prime}\right)\right] e^{j n \phi^{\prime}}
$$

Similarly, the scattered tangent electric field evaluated at the surface is found by using (12) and (13) in (14)

$$
\begin{aligned}
E_{\text {tan }}^{s}= & \frac{1}{j \omega_{e} \epsilon} \sum_{n=-\infty}^{\infty} j^{-n} \frac{J_{n}^{\prime}(k a)}{H_{n}^{(2) \prime}(k a)}\left(1+k b D_{n}\right) \alpha \\
& \times\left[\beta \frac{j n}{\rho^{\prime}} H_{n}^{(2)}\left(k \rho^{\prime}\right)-k H_{n}^{(2) \prime}\left(k \rho^{\prime}\right)\right] e^{j n \phi^{\prime}} .
\end{aligned}
$$

We will use Taylor series expansion to approximate the derivatives of the eigenfunctions in (15) and (16). To the first order in $k b$

$$
Z_{n}^{\prime}\left(k \rho^{\prime}\right)=Z_{n}^{\prime}\left(k\left(a+b f\left(\phi^{\prime}, t\right)\right) \approx Z_{n}^{\prime}(k a)+k b f\left(\phi^{\prime}, t\right) Z_{n}^{\prime \prime}(k a)\right.
$$

where as before $Z_{n}$ represents either the Bessel or Hankel function. Using the approximations of (5) and (17) in (15) and (16) and applying the boundary condition at the surface of the cylinder leads to

$$
\begin{aligned}
& \sum_{n=-\infty}^{\infty} j^{-n} J_{n}^{\prime}(k a) D_{n} e^{j n \phi^{\prime}} \\
&=\sum_{n=-\infty}^{\infty} j^{-n}\left[f\left(\phi^{\prime}, t\right) \frac{W_{n}}{H_{n}^{(2) \prime}(k a}\right. \\
&\left.-f^{\prime}\left(\phi^{\prime}, t\right) \frac{2 n}{\pi(k a)^{3} H_{n}^{(2) \prime}(k a)}\right] e^{j n \phi^{\prime}}
\end{aligned}
$$


where $W_{n}=J_{n}^{\prime \prime}(k a) H_{n}^{(2)^{\prime}}(k a)-J_{n}^{\prime}(k a) H_{n}^{(2)^{\prime \prime}}(k a)$, and the $(k b)^{2}$ and higher terms have been neglected. Expressing $f\left(\phi^{\prime}, t\right)$ in terms of its Fourier series expansion, $f^{\prime}\left(\phi^{\prime}, t\right)$ can be written as

$$
f^{\prime}\left(\phi^{\prime}, t\right)=\sum_{m=-\infty}^{\infty} j m A_{m}(t) e^{j m \phi^{\prime}}
$$

which can be inserted in (18) to provide the solution for the perturbation coefficients

$$
D_{n}(t)=\frac{j^{n}}{J_{n}^{\prime}(k a)} \sum_{p=-\infty}^{\infty} j^{-p} \frac{A_{n-p}(t)}{H_{p}^{(2) \prime}(k a)}\left[W_{p}-\frac{j 2(n-p) p}{\pi(k a)^{3}}\right] .
$$

Thus for a given displacement function, $f\left(\phi^{\prime}, t\right)$, the $D_{n}^{\prime} \mathrm{s}$ found from (19) can be used in (12) to give the time-varying scattered field at any angle $\phi$. As before, the Fourier transform of this time-varying field provides the scattered Doppler spectrum.

\section{Acoustic Vibration OF A SOLID Cylinder}

\section{A. Mathematical Formulation for Displacement}

In order to calculate the deformation of a circular cylinder due to a time-harmonic incident acoustic wave, the acoustic scattering from the cylinder must be evaluated. An interesting aspect of acoustic scattering from solid objects is that mechanical waves within the solid are excited which consist of both transverse shear waves and longitudinal compressional waves. Both of these components must be accounted for in the solid to accurately describe the deformation. The fluid medium surrounding the cylinder is assumed to be nonviscous and supports only compressional waves. A thorough treatment of the acoustic scattering of an incident plane wave from a solid cylinder is given in [16]. Inherent in this formulation is the resulting mechanical vibration of the cylinder caused by the incident acoustic wave which is of interest to us.

The procedure for evaluating the scattering of an acoustic plane wave by a solid cylinder includes finding suitable solutions to the equation of motion of a solid elastic medium, defining the wave motion outside the scatterer to consist of an incident acoustic plane wave and an outgoing scattered wave, and applying the proper boundary conditions at the surface of the cylinder. The equation of motion for a solid elastic medium can be written [17]

$$
\frac{E(1-\sigma)}{(1+\sigma)(1-2 \sigma)} \nabla(\nabla \cdot \mathbf{u})-\frac{E}{2(1+\sigma)} \nabla \times(\nabla \times \mathbf{u})=m_{1} \frac{\partial^{2} \mathbf{u}}{\partial t^{2}}
$$

where $m_{1}$ is the density of the scatterer, $\mathbf{u}$ is the displacement, and $E$ and $\sigma$ are Young's modulus and Poisson's ratio, respectively. Solutions to this equation for a solid cylinder of infinite length are of the form [16]

$$
\begin{aligned}
& u \rho=\sum_{n=0}^{\infty}\left[\frac{n b_{n}}{\rho} J_{n}\left(\kappa_{2} \rho\right)-a_{n} \frac{d}{d_{\rho}} J_{n}\left(\kappa_{1} \rho\right)\right] \cos n \phi \\
& u \phi=\sum_{n=0}^{\infty}\left[\frac{n a_{n}}{\rho} J_{n}\left(\kappa_{1} \rho\right)-b_{n} \frac{d}{d \rho} J_{n}\left(\kappa_{2} \rho\right)\right] \sin n \phi
\end{aligned}
$$

where $\kappa 1=\omega_{a} / c_{1}$ and $\kappa_{2}=\omega_{a} / c_{2}$ are propagation constants for the compressional wave and shear wave within the scatterer, respectively, and $a_{n}$ and $b_{n}$ must be determined from the boundary conditions. The velocity of the compressional wave is

$$
c_{1}=\sqrt{\frac{E(1-\sigma)}{m_{1}(1+\sigma)(1-2 \sigma)}}
$$

and the shear wave velocity is

$$
c_{2}=\sqrt{\frac{E}{2 m_{1}(1+\sigma)}}
$$

The incident acoustic plane wave propagating in the surrounding nonviscous fluid medium is represented by

$$
\begin{aligned}
p_{i} & =P_{0} e^{-j \kappa_{3} x}=P_{0} e^{-j \kappa_{3} \rho \cos \phi} \\
& =P_{0} \sum_{n=0}^{\infty} \epsilon_{n}(-j)^{2} J_{n}\left(\kappa_{3} \rho\right) \cos n \phi
\end{aligned}
$$

and the scattered acoustic wave is

$$
p_{s}=\sum_{n=0}^{\infty} c_{n} H_{n}^{2}\left(\kappa_{3} \rho\right) \cos n \phi
$$

where $p$ represents the pressure variation, $\kappa_{3}$ is the propagation constant for the surrounding fluid medium, and $c_{n}$ must be determined from the boundary conditions. The radial components of displacement associated with the incident and scattered waves are

$$
u_{i, p}=\frac{1}{m_{3} \omega_{a}^{2}} \frac{\partial p_{i}}{\partial \rho} \quad \text { and } \quad u_{s, p}=\frac{1}{m_{3} \omega_{a}^{2}} \frac{\partial p_{s}}{\partial \rho}
$$

where $m_{3}$ is the density of the surrounding medium. Applying three boundary conditions provides solutions for $a_{n}, b_{n}$, and $c_{n}$, whereupon $a_{n}$ and $b_{n}$ are used in (21) and (22) to give the radial and angular displacements of the cylinder.

The following boundary conditions are applied at the surface of the cylinder:

i) the pressure in the fluid must equal the normal component of stress in the solid;

ii) the normal component of displacement in the fluid must equal the normal displacement of the solid;

iii) the tangential components of shearing stress must vanish at the surface of the solid.

Explicitly

$$
\begin{aligned}
p_{i}+p_{s} & =-[\rho \rho] \quad \text { at } \quad \rho=a \\
u_{i, p}+u_{s, p} & =u_{\rho} \quad \text { at } \quad \rho=a \\
{[\rho \phi]=[\rho z] } & =0 \quad \text { at } \quad \rho=a
\end{aligned}
$$

with

$$
\begin{aligned}
& {[\rho \rho]=\frac{E \sigma}{(1+\sigma)(1-2 \sigma)} \nabla \cdot \mathbf{u}+\frac{E}{1+\sigma} \frac{\partial u_{p}}{\partial \rho}} \\
& {[\rho \phi]=\frac{E}{2(1+\sigma)}\left[\frac{1}{\rho} \frac{\partial u_{p}}{\partial \phi}+\rho \frac{\partial}{\partial \rho} \frac{u_{\phi}}{\rho}\right]} \\
& {[\rho z]=\frac{E}{2(1+\sigma)}\left[\frac{\partial u_{p}}{\partial z}+\frac{\partial u_{z}}{\partial \rho}\right] .}
\end{aligned}
$$


Upon applying these conditions, solving the resulting system of equations for $a_{n}$ and $b_{n}$ is tedious but straightforward. The results are given in the Appendix. It should be noted from the solution of $a_{n}$ and $b_{n}$ that a linear relationship exists between the displacements in (21) and (22) and the amplitude, $P_{o}$, of the incident pressure wave.

A relevant topic to consider at this point is the normal modes of free (unforced) vibration in the scattering body. The normal modes of free vibration occur at frequencies that yield a nontrivial solution to the homogeneous scattering problem where no external forces exist. The boundary conditions for free vibration are simply that

i) the normal component of stress;

ii) the tangential component of shearing stress must both vanish at the surface of the body.

These conditions are identical to the first and third conditions in the original scattering problem with the incident and scattered amplitudes set to zero. It can be shown that these conditions hold at frequencies satisfying

$$
A_{2} B_{3}+B_{2} A_{3}=0
$$

where $A_{2}, A_{3}, B_{2}$, and $B_{3}$ are defined in the Appendix. As discussed later, an incident acoustic wave at one of these resonant frequencies produces a significant displacement for that mode. Conversely, if the incident acoustic frequency is far from a resonance of the cylinder, then negligible displacement occurs. This important property is the basis for the proposed target detection/identification scheme in a strong clutter environment where the Doppler spectrum of the electromagnetic scattered field from an object vibrating at its natural resonances is used for discrimination. Also, note from (29) that the mechanical resonant frequencies of the object depend only on the mechanical properties of the object and are independent of the surrounding medium. The frequencies at which maximum displacement occurs may shift, however, if the density contrast between the object and background medium is changed.

\section{B. Mapping to Radial Displacement}

The displacement of the cylinder is now represented by both a radial and angular displacement in (21) and (22). To apply the perturbation solution for electromagnetic scattering given in Section II, the displacement must be expressed by only radial displacement as a function of angle, and ultimately, must be written as a Fourier series in $\phi^{\prime}$. Recall that the perimeter of the cylinder is expressed from (1) as $\rho^{\prime}=a+b f\left(\phi^{\prime}, t\right)$ with the radial displacement given by $b f\left(\phi^{\prime}, t\right)$. The radial and angular displacement can be mapped into only radial displacement by setting

$$
b f\left(\phi^{\prime}, t\right)=u_{\rho}\left(\phi^{\prime}-\phi_{o}, t\right)
$$

where $\phi_{o}$ is the angular shift corresponding to the angular component of displacement, $u_{\phi}$. From basic geometry (see Fig. 3) and an application of Taylor series about $\phi_{o}$

$$
\phi_{o}=\frac{u_{\phi}\left(\phi^{\prime}-\phi_{o}, t\right)}{a} \approx \frac{u_{\phi}\left(\phi^{\prime}, t\right)}{a}-\phi_{o} \frac{u_{\phi}^{\prime}\left(\phi^{\prime}, t\right)}{a} .
$$

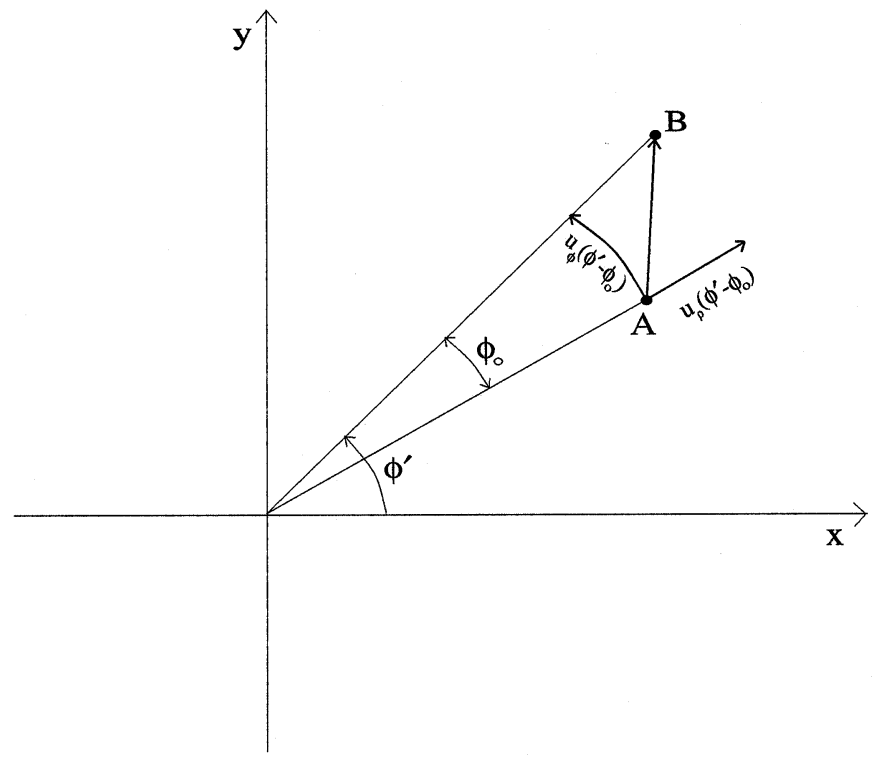

Fig. 3. Mapping the displacement from point $\mathrm{A}\left(\rho^{\prime}=a\right)$ to point $\mathrm{B}\left(\rho^{\prime}=\right.$ $\left.a+b f\left(\phi^{\prime}\right)\right)$.

Solving for $\phi_{o}$

$$
\phi_{o}=\frac{u_{\phi}\left(\phi^{\prime}, t\right)}{a+u_{\phi}^{\prime}\left(\phi^{\prime}, t\right)} \approx \frac{u_{\phi}\left(\phi^{\prime}, t\right)}{a}
$$

since $u_{\phi}^{\prime}\left(\phi^{\prime}, t\right) \ll a$. And substituting (32) into (30) gives

$$
b f\left(\phi^{\prime}, t\right)=u_{\rho}\left(\phi^{\prime}-\frac{u_{\phi}\left(\phi^{\prime}, t\right)}{a}, t\right) .
$$

Rewriting $u_{\rho}$ and $u_{\phi}$ with the time dependence explicitly shown

$$
\begin{aligned}
& u_{\rho}\left(\phi^{\prime}, t\right)=\sum_{n=0}^{\infty} U_{\rho, n} \cos \left(\omega_{a} t+\theta_{\rho, n}\right) \cos n \phi^{\prime} \\
& u_{\phi}\left(\phi^{\prime}, t\right)=\sum_{n=0}^{\infty} U_{\phi, n} \cos \left(\omega_{a} t+\theta_{\phi, n}\right) \sin n \phi^{\prime}
\end{aligned}
$$

where $U_{\rho, n}$ and $U_{\phi, n}$ are the magnitudes of the coefficients for the $n^{\text {th }}$ mode given by the expressions in brackets in (21) and (22), respectively, and $\theta_{\rho, n}$ and $\theta_{\phi, n}$ are the respective phases of these coefficients. Substituting (34)and (35) into (33) yields

$$
\begin{aligned}
& b f\left(\phi^{\prime}, t\right)= \sum_{n=0}^{\infty} U_{\rho, n} \cos \left(\omega_{a} t+\theta_{\rho, n}\right) \\
& \cos \left[n \left(\phi^{\prime}-\frac{\sum_{m=0}^{\infty} U_{\phi, m} \cos \left(\omega_{a} t+\theta_{\phi, m}\right)}{a}\right.\right. \\
&\left.\left.\sin m \phi^{\prime}\right)\right] .
\end{aligned}
$$

In general, this expression should be expanded in a Fourier series about $\phi^{\prime}$. The coefficients, $A_{m}(t)$, of the expansion can then be used in the TM or TE electromagnetic solution to obtain the time-varying scattered field. When only one mode has significant displacement, however, (36) for the $n^{\text {th }}$ mode simplifies to

$$
\begin{aligned}
b f\left(\phi^{\prime}, t\right)= & U_{\rho, n} \cos \left(\omega_{a} t+\theta_{\rho, n}\right) \\
& \cos \left[n\left(\phi^{\prime}-\frac{U_{\phi, n} \cos \left(\omega_{a} t+\theta_{\phi, n}\right)}{a} \sin n \phi^{\prime}\right)\right] .
\end{aligned}
$$




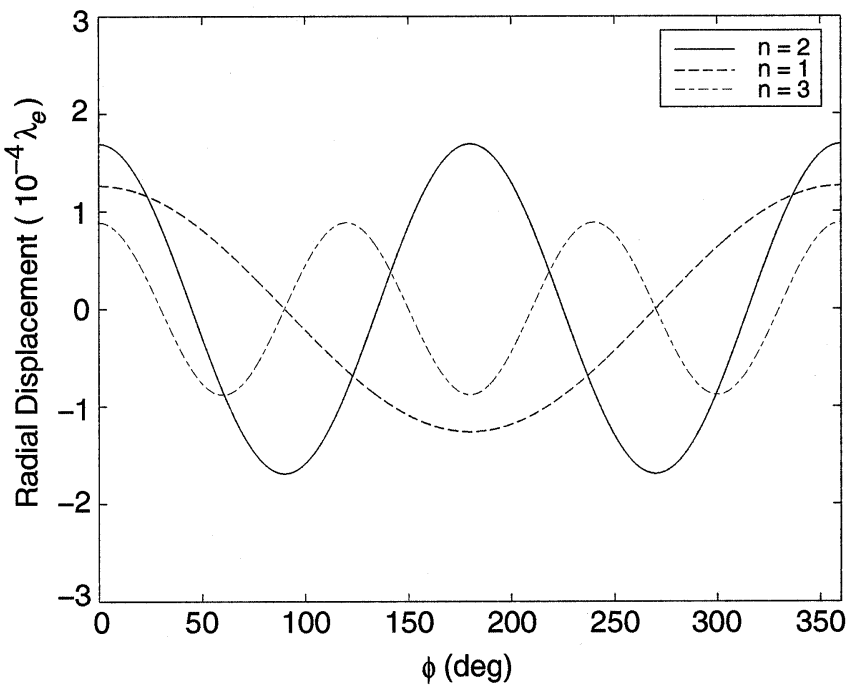

Fig. 4. Peak surface displacement for the first three resonant mechanical modes of a copper cylinder. The radius, $a$, of the cylinder is $\lambda_{e}$.

Taking $b=U_{\rho, n}$, we can expand $f\left(\phi^{\prime}, t\right)$ in a Fourier series about $\phi^{\prime}$. The coefficients, $A_{m}(t)$, of the expansion are conveniently written in terms of Bessel functions as [18], see (38), shown at the bottom of the page. This completes the solution for a given mode of vibration in the cylinder. The above coefficients should be substituted into (9) or (19) for the TM or TE case, respectively, to solve for the perturbation coefficients, followed by a substitution into (4) or (12) for the electromagnetic scattering solution. As stated before, the Fourier transform of this solution gives the scattered Doppler spectrum.

\section{Simulations}

When the frequency of the incident acoustic wave is not close to a resonant frequency of the cylinder, it behaves as an impenetrable rigid cylinder [19]. Consequently, there is negligible displacement at the surface of the cylinder, and the magnitude of the Doppler spectrum is also negligible. However, when the frequency of the incident acoustic wave is close to a resonance of the object, the mode corresponding to this resonance is excited and a measurable Doppler spectrum results.

\section{A. Excitation at Resonance}

As an example, consider a $\lambda_{e}$ (wavelength of electromagnetic wave) radius, solid copper cylinder illuminated by an incident acoustic plane wave in air with a power density of $1 \mathrm{~kW} / \mathrm{m}^{2}$. The peak displacement for the first three resonances of a solid copper cylinder are shown in Fig. 4. Recall that the displacement is linearly related to the amplitude of the incident acoustic wave and can be scaled accordingly. Now let the frequency of the incident acoustic wave correspond to the first resonance of

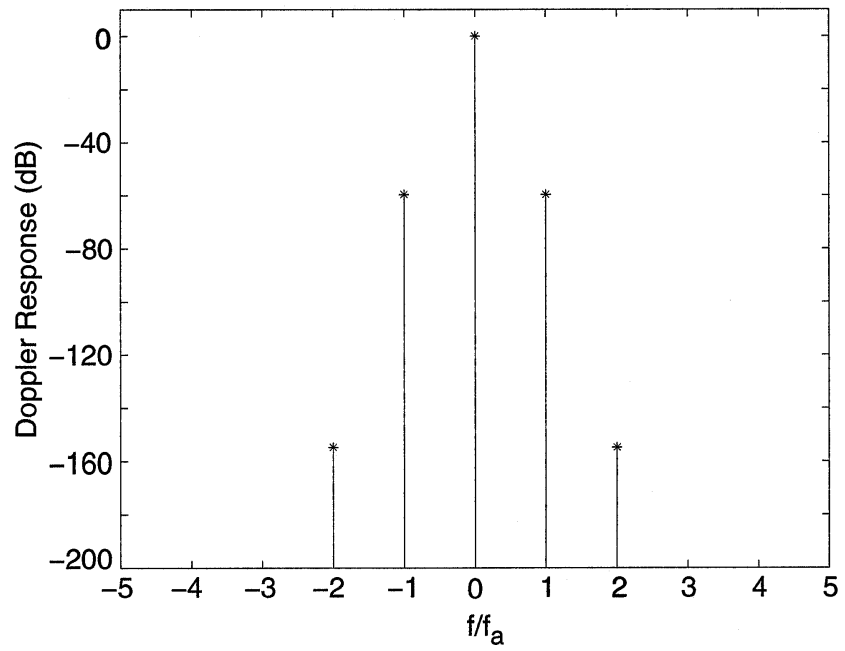

Fig. 5. Backscattered Doppler spectrum of a TM incident plane wave from an acoustically vibrated, $\lambda_{e}$ radius, copper cylinder. The second mechanical mode is excited $(n=2)$.

the $n=2$ mode. This frequency is found by solving (29) and occurs when $\kappa_{1} a=1.174$. The Young's Modulus, $E$, for copper is about $127 \mathrm{GPa}$, and Poisson's ratio, $\sigma$, is taken to be $1 / 3$, which results in a resonance at $f_{a}=864.5 / a \mathrm{~Hz}$ for the $n=2$ mode of the solid copper cylinder. Fig. 5 shows the calculated Doppler spectrum of the backscatter with TM incidence for this mode. The spectrum, similar to a frequency modulated signal with a low index of modulation, has frequency components at harmonics of the incident acoustic frequency of which the first is by far the largest. Other acoustic modes produce a similar Doppler spectrum where the frequency components are at harmonics of the vibration frequency and the first component is still the most significant. These results apply for both TM and TE incidence and suggest that the frequency of vibration of the scatterer can be determined by the first harmonic of the scattered Doppler spectrum. Although the first harmonic is the largest, it is still considerably lower $(60 \mathrm{~dB})$ than the carrier frequency and a sensitive measuring system must be used. To illustrate the system requirements, consider a $5 \mathrm{~cm}$ radius, copper cylinder with a first mechanical resonance at $f_{a}=17.29 \mathrm{kHz}$. The phase noise at this frequency must be below approximately $-60 \mathrm{dBc} / \mathrm{Hz}$ at $f_{a}$ to measure the first harmonic of Doppler spectrum. Since oscillators with phase noise of $-100 \mathrm{dBc} / \mathrm{Hz}$ at $10 \mathrm{kHz}$ can easily be made, accurate measurement of the Doppler spectrum is indeed feasible.

To illustrate the effect of resonance on the scattered Doppler spectrum, the magnitude of the first harmonic of the Doppler spectrum in the backscatter is calculated as a function of the incident acoustic frequency. Fig. 6 shows that the first harmonic of the scattered Doppler spectrum for the copper cylinder in air is highly sensitive to resonances in the cylinder. In fact,

$$
A_{m}(t)=\cos \left(\omega_{a} t+\theta_{\rho}\right) \begin{cases}1 & \text { for } m=0, n=0 \\ \frac{1}{2}\left[J_{1+\frac{m}{n}}\left(\frac{n U_{\phi, n} \cos \left(\omega_{a} t+\theta_{\phi, n}\right)}{a}\right)+J_{1-\frac{m}{n}}\left(\frac{n U_{\phi, n} \cos \left(\omega_{a} t+\theta_{\phi, n}\right)}{a}\right)\right] & \text { for } m / n \text { an integer, } n \neq 0 \\ 0 & \text { otherwise }\end{cases}
$$




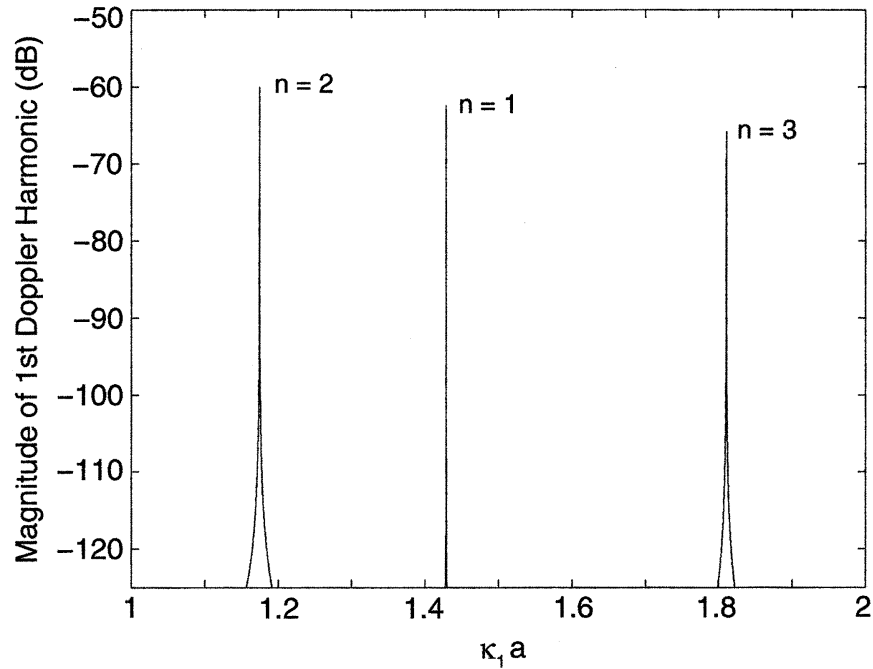

Fig. 6. Variation of the first Doppler harmonic magnitude with acoustic frequency for a copper cylinder in air. $\kappa_{1}=\omega_{a} / c_{1}$ is the propagation constant for the compressional wave within the cylinder. For a $5 \mathrm{~cm}$ radius cylinder, the acoustic frequency scan shown here is from $f_{a}=14.7 \mathrm{kHz}$ to $f_{a}=29.4 \mathrm{kHz}$

the scattered Doppler components are negligible except around narrow resonances corresponding to the resonant frequencies of the cylinder. Note that the $n=2$ mode is resonant at the lowest frequency, followed by the $n=1$ and $n=3$ modes. Hence, by acoustically vibrating the cylinder over a wide range of frequencies, the Doppler response will have significant Doppler components only at the mechanical resonant frequencies of the cylinder. This technique could be used in general to measure the mechanical resonances of any object. Furthermore, using the Doppler backscatter to measure these resonances could provide a means of object discrimination based on the location and distribution of the resonances in the Doppler response.

It should be noted that when the density of the background medium is increased (or, equivalently, the density contrast between the object and background is decreased), the magnitude of resonant vibration in the object, and consequently the magnitude of the Doppler response, is reduced. Since the displacements of solid metallic objects are small in an air background, the displacement in a dense medium (such as soil) could be below the sensitivity of the measuring instrument. This undesirable result is offset by realizing that in a practical scenario the buried objects of interest are likely to be metallic or plastic shells which will have significantly larger displacements (and lower resonant frequencies) than the virtually rigid, solid objects considered here. To fully extend the present analysis to a soil background, the complex wave structure of mechanical waves in soil such as the compressional, shear, and surface (Rayleigh) waves must be considered and is left for future work.

\section{B. Bistatic Scattering of Doppler Spectrum}

A natural extension to the Doppler backscatter is the variation of the Doppler scattering as a function of angle, $\phi$. The Doppler scattering (TM incidence) from a copper cylinder excited at its first three mechanical resonances corresponding to the $n=2, n=1$, and $n=3$ modes, respectively, is simulated with $\phi$ varying from $0^{\circ}$ (forward scatter) to $180^{\circ}$ (backscatter).

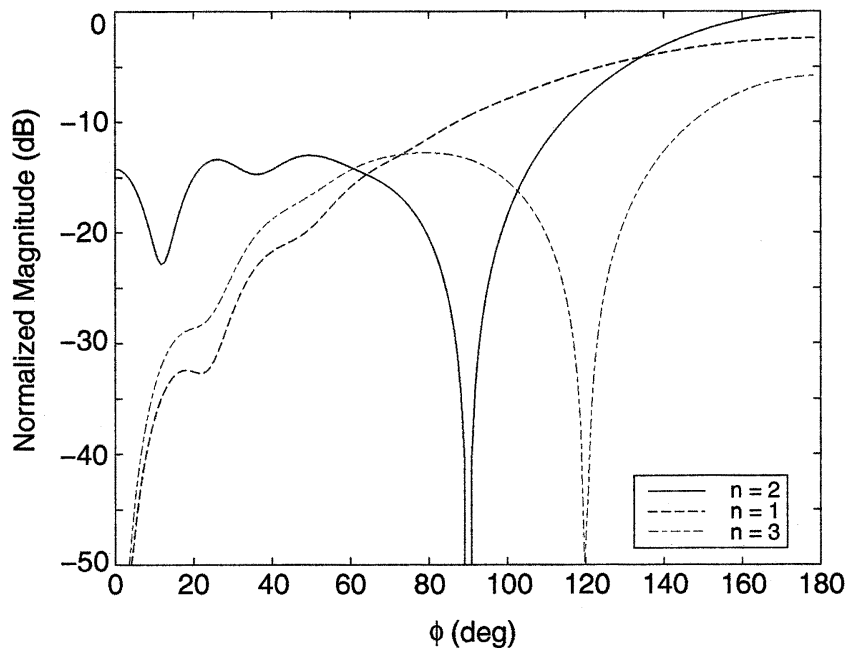

Fig. 7. Variation of scattered first Doppler harmonic magnitude with $\phi$ for TM incidence. $\left(a=\lambda_{e}\right)$.

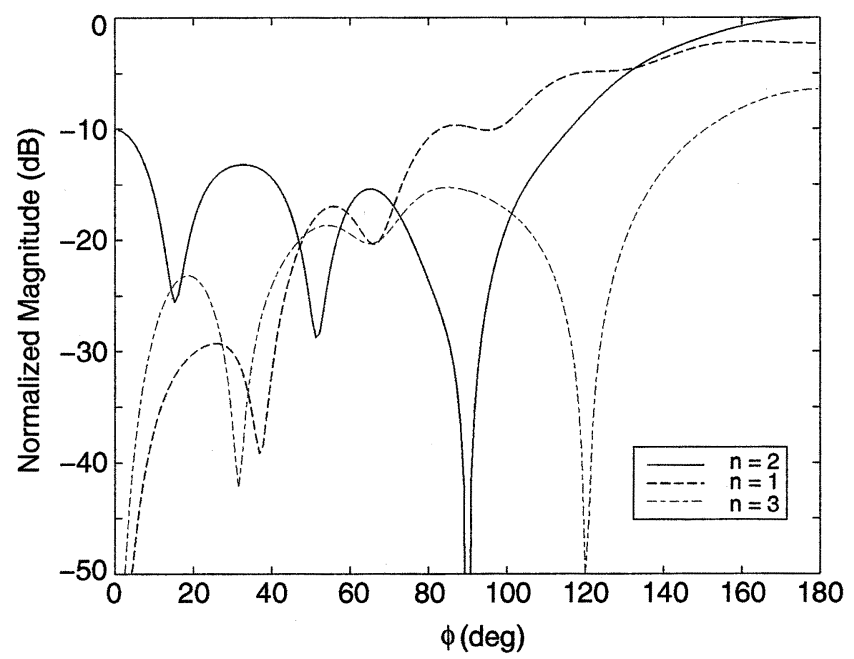

Fig. 8. Variation of scattered first Doppler harmonic magnitude with $\phi$ for TE incidence. $\left(a=\lambda_{e}\right)$.

A plot of the first harmonic component for each mode versus scattering angle is given in Fig. 7. One significant observation is that the location of the nulls, and the angular response in general, depends strongly on the excited mode(s) of the object. The nulls occur at angles where the symmetry of the bistatic scattering for a particular mode produces no net Doppler shift. The bistatic variation of the first harmonic of the Doppler spectrum for TE incidence is shown in Fig. 8. Note that the angles of the nulls are identical to the TM case, and the overall angular response follows a similar trend.

\section{CONCLUSION}

In this paper, the analytical Doppler spectrum of an acoustically vibrated circular cylinder is examined and shown to provide a measurable quantity which is strongly dependent upon the cylinder's mechanical resonances. A perturbation method is employed to derive an analytical solution for the 
bistatic scattering from a deformed cylinder. Using the solution for the displacement of a solid cylinder with acoustic excitation, the Doppler spectrum scattered from the vibrating cylinder is obtained. The Doppler spectrum is shown to consist of harmonics of the incident acoustic frequency where the first harmonic is the most significant. Also, the first harmonic only becomes measurable when the cylinder is excited near a mechanical resonance. Thus, by sweeping the frequency of acoustic excitation, the resulting Doppler spectrum will be dominated by frequency components corresponding to the mechanical resonances of the cylinder. The location of these resonances does not depend strongly upon the background medium. Furthermore, the scattered Doppler magnitude for a given mode is shown to be a strong function of scattering angle. These results indicate that acoustically vibrating an object at its resonant frequencies and measuring the Doppler scattered response monostatically or bistatically could provide an effective method for detecting and identifying buried objects.

\section{APPENDIX}

The coefficients for displacement used in Section III are given here and can be substituted into (21) and (22) for the radial and angular displacements, respectively.

$$
\begin{aligned}
a_{n}= & \frac{2 P_{o} \epsilon_{n} j^{-n+1} a^{2}}{\pi \omega_{a}^{2} \rho_{3}} \\
& \cdot \frac{1}{C_{1}\left(A_{2}+\frac{B_{2} A_{3}}{B_{3}}\right)-C_{2}\left(A_{1}+\frac{B_{1} A_{3}}{B_{3}}\right)} \\
b_{n}= & \frac{A_{3}}{B_{3}} a_{n}
\end{aligned}
$$

where

$$
\begin{aligned}
& A_{1}=\kappa_{1} a J_{n}^{\prime}\left(\kappa_{1} a\right) \\
& B_{1}=-n J_{n}\left(\kappa_{2} a\right) \\
& C_{1}=\frac{\kappa_{3} a}{\left(\omega_{a}^{2} \rho_{3}\right) H_{n}^{(2) \prime}\left(\kappa_{3} a\right)} \\
& A_{2}=2 \rho_{1} c_{2}^{2}\left(\kappa_{1} a\right)^{2}\left[\frac{\sigma}{(1-2 \sigma) J_{n}\left(\kappa_{1} a\right)-J_{n}^{\prime \prime}\left(\kappa_{1} a\right)}\right] \\
& B_{2}=2 \rho_{1} c_{2}^{2} n\left[\kappa_{2} a J_{n}^{\prime}\left(\kappa_{2} a\right)-J_{n}\left(\kappa_{2} a\right)\right] \\
& C_{2}=a^{2} H_{n}^{(2)}\left(\kappa_{3} a\right) \\
& A_{3}=2 n\left[\kappa_{1} a J_{n}^{\prime}\left(\kappa_{1} a\right)-J_{n}\left(\kappa_{1} a\right)\right] \\
& B_{3}=n^{2} J_{n}\left(\kappa_{2} a\right)-\kappa_{2} a J_{n}^{\prime}\left(\kappa_{2} a\right)+\left(\kappa_{2} a\right)^{2} J_{n}^{\prime \prime}\left(\kappa_{2} a\right) .
\end{aligned}
$$

\section{REFERENCES}

[1] S. Koppenjan et al., "Ground penetrating radar applications: Dept. of Energy case studies," in Proc. SPIE—Int. Soc. Opt. Eng., vol. 1942, 1993, pp. $145-153$.

[2] C. Stewart, "Summary of Mine Detection Research," U.S. Army Eng. R\&D Labs, Belvoir, VA, Tech. Rep. 1636-TR, vol. I, May 1960.

[3] G. S. Smith, "Workshop on new directions for electromagnetic detection of non-metallic mines," Tech. Rep., BRDEC and ARO, U.S. Army, June 1992.

[4] W. R. Scott Jr. and J. S. Martin, "An experimental model of a acoustoelectromagnetic sensor for detecting land mines," in Proc. IEEE AP-S Int. Symp., Atlanta, GA, July 1998, pp. 978-981.
[5] L. Rayleigh, The Theory of Sound. New York: Dover, 1945, vol. II, p. 89.

[6] J. C. Maxwell, A Treatise on Electricity and Magnetism. New York: Dover, 1954, vol. I, p. 220

[7] S. O. Rice, "Reflection of electromagnetic waves by slightly rough surfaces," in The Theory of Electromagnetic Waves, M. Kline, Ed. New York: Intersciences, 1963.

[8] G. S. Agarwal, "Interaction of electromagnetic waves at rough dielectric surfaces," Phys. Rev. B, vol. 15, pp. 2371-2383, 1977.

[9] P. C. Clemmow and V. H. Weston, "Diffraction of a plane wave by an almost circular cylinder," in Proc. Roy. Soc. London A, vol. 264, 1961, p. 246.

[10] C. Yeh, "Perturbation method in the diffraction of electromagnetic waves by arbitrary shaped penetrable objects," J. Math. Phys., vol. 6, no. 12, pp. 2008-2013, Dec. 1965.

[11] K. M. Mitzner, "Effect of small irregularities on electromagnetic scattering from an interface of arbitrary shape," J. Math. Phys., vol. 5, no. 12, pp. 1776-1786, Dec. 1964.

[12] C. Yeh, "Perturbation approach to diffraction of electromagnetic waves by arbitrarily shaped dielectric obstacles," Phys. Rev. A, vol. 135, p. 1193,1964

[13] M. L. Burrows, "A reformulated boundary perturbation theory in electromagnetism and its application to a sphere," Canadian J. Phys., vol. 45, pp. 1729-1743, 1967.

[14] D. E. Lawrence and K. Sarabandi, "Acoustic and electromagnetic wave interaction: analytical formulation for acousto-electromagnetic scattering behavior of a dielectric cylinder," IEEE Trans. Antennas Propagat., vol. 49, pp. 1382-1392, Oct. 2001.

[15] R. F. Harrington, Time-Harmonic Electromagnetic Fields. New York: McGraw-Hill, 1961, pp. 232-235.

[16] J. J. Faran, "Sound scattering by solid cylinders and spheres," J. Acous. Soc. Amer, vol. 23, no. 4, pp. 405-418, July 1951.

[17] A. E. H. Love, A Treatise on the Mathematical Theory of Elasticity, 4th ed. New York: Dover, 1944.

[18] F. E. Relton, Applied Bessel Functions. New York: Dover, 1965, pp. $153-155$.

[19] W. P. Mason and R. N. Thurston, Physical Acoustics Principles and Methods. New York: Academic, 1981, vol. XV, pp. 191-202.

Kamal Sarabandi (S'87-M'90-SM'92-F'00) received the B.S. degree in electrical engineering from the Sharif University of Technology, Tehran, Iran, in 1980, and the M.S. degree in electrical engineering, the M.S. degree in mathematics, and the Ph.D. degree in electrical engineering from the University of Michigan, Ann Arbor, in 1980, 1986, and 1989, respectively.

$\mathrm{He}$ is the Director of the Radiation Laboratory and a Professor in the Department of Electrical Engineering and Computer Science, University of Michigan. His research areas of interest include microwave and millimeter-wave radar remote sensing, electromagnetic wave propagation, and antenna miniaturization. He has 20 years of experience with wave propagation in random media, communication channel modeling, microwave sensors, and radar systems and is leading a large research group including two research scientists, 10 Ph.D. and two M.S. students. He has served as the Principal Investigator on many projects sponsored by NASA, JPL, ARO, ONR, ARL, NSF, DARPA, and numerous industries. He has published many book chapters and more than 95 papers in refereed journals on electromagnetic scattering, random media modeling, wave propagation, antennas, microwave measurement techniques, radar calibration, inverse scattering problems, and microwave sensors. He has also had more than 200 papers and invited presentations in many national and international conferences and symposia on similar subjects.

Dr. Sarabandi received the prestigious Henry Russel Award from the Regent of The University of Michigan, the GAAC Distinguished Lecturer Award from the German Federal Ministry for Education, Science, and Technology, in 1999, and the 1996 Teaching Excellence Award from the EECS Department of The University of Michigan. He is listed in American Men \& Women of Science Who's Who in America and Who's Who in Electromagnetics. He is a Vice President of the IEEE Geoscience and Remote Sensing Society (GRSS), Chairman of the Awards Committee of the IEEE GRSS, and a member of IEEE Technical Activities Board Awards Committee. He is serving as the Associate Editor of the IEEE TRANSACTIONS ON ANTENNAS AND PROPAGATION (AP) and the IEEE SENSORS JOURNAL. He is also a member of Commission F of URSI and of The Electromagnetic Academy. 
Daniel E. Lawrence (S'96-M'02) was born in Silver Spring, MD, on June 8, 1973. He received the B.E.E. degree and M.S. degrees in electrical engineering from Auburn University, Auburn, AL, in 1996 and 1998, respectively, and is currently pursuing the Ph.D. degree in the Radiation Laboratory, University of Michigan, Ann Arbor.

From 1997 to 2000, he was a DOD Graduate Research Fellow investigating the use of low-frequency magnetic fields for buried landmine discrimination. He has also performed analytical analysis of acousto-electromagnetic methods applied to buried object detection. His current research interests include microwave and millimeter-wave radar systems, techniques used in buried landmine detection/identification, and the interaction of acoustic and electromagnetic waves. 